\title{
TAK1 inhibition-induced RIP1-dependent apoptosis in murine macrophages relies on constitutive TNF-a signaling and ROS production
}

\author{
Jang-Shiun Wang ${ }^{1,2+}$, Dean Wü ${ }^{3 \dagger}$, Duen-Yi Huang ${ }^{1}$ and Wan-Wan Lin ${ }^{1,2^{*}}$
}

\begin{abstract}
Background: Transforming growth factor- $\beta$ (TGF- $\beta$ )-activated kinase 1 (TAK1) is a key regulator of signal cascades of TNF-a receptor and TLR4, and can induce NF-KB activation for preventing cell apoptosis and eliciting inflammation response.

Results: TAK1 inhibitor (TAKI) can decrease the cell viability of murine bone marrow-derived macrophages (BMDM), RAW264.7 and BV-2 cells, but not dermal microvascular endothelial cells, normal human epidermal keratinocytes, THP-1 monocytes, human retinal pigment epithelial cells, microglia CHME3 cells, and some cancer cell lines (CL1.0, HeLa and HCT116). In BMDM, TAKI-induced caspase activation and cell apoptosis were enhanced by lipopolysaccharide (LPS). Moreover, TAKI treatment increased the cytosolic and mitochondrial reactive oxygen species (ROS) production, and ROS scavengers NAC and BHA can inhibit cell death caused by TAKI. In addition, RIP1 inhibitor (necrostatin-1) can protect cells against TAKI-induced mitochondrial ROS production and cell apoptosis. We also observed the mitochondrial membrane potential loss after TAKI treatment and deterioration of oxygen consumption upon combination with LPS. Notably TNF-a neutralization antibody and inhibitor enbrel can decrease the cell death caused by TAKI.
\end{abstract}

Conclusions: TAKl-induced cytotoxicity is cell context specific, and apoptosis observed in macrophages is dependent on the constitutive autocrine action of TNF-a for RIP1 activation and ROS production.

Keywords: TAK1, RIP1, Macrophages, TNF-a, ROS, Apoptosis

\section{Background}

Transforming growth factor- $\beta$ (TGF- $\beta$ )-activated kinase 1 (TAK1) is a ubiquitously expressed mitogen-activated protein kinase kinase kinase and plays a key role in regulating inflammation, immunity, cell differentiation and death [1-3]. Accumulating evidence indicates that TAK1 is a key regulator of signal transduction cascades and is activated by various inflammatory mediators and cytokines such as transforming growth factor (TGF)- $\beta$, tumor necrosis factor (TNF)- $\alpha$, interleukin (IL)-1 $\beta$, CD40 ligand, toll-like receptor (TLR) ligands, $\mathrm{T}$ and $\mathrm{B}$ cell receptor

\footnotetext{
* Correspondence: wwllaura1119@ntu.edu.tw

${ }^{\dagger}$ Equal contributors

'Department of Pharmacology, College of Medicine, National Taiwan University, No 1, Sec 1, Jen-Ai Road, Taipei, Taiwan

${ }^{2}$ Graduate Institute of Medical Sciences, Taipei Medical University, Taipei, Taiwan

Full list of author information is available at the end of the article
}

ligands $[3,4]$. TAK1 activity is tightly regulated by its binding proteins, TAB1 and TAB2/TAB3, as well as by posttranslational modification including ubiquitination and phosphorylation. In TLR4 signaling, TRAF6 through its E3 ubiquitin ligase activity facilitates the formation of $\mathrm{K} 63$ polyubiquitin chains to recruit and activate TAK1 [5]. This activation then transduces signals for activating downstream kinases IKK, p38, and JNK, in turn leading to activate NF- $\mathrm{kB}$ and activator protein-1 (AP-1) to produce the proinflammatory and anti-apoptosis proteins $[4,6-8]$. Consistently the notion implicating TAK1 as a key intermediary in cell survival is evidenced by observing the embryonic lethality associated with multi-tissue defects in germline deficient of TAK1 gene [4]. Subsequent studies in mice with the conditional ablation of TAK1 in hepatocytes [9], liver parenchymal cells [10], keratinocytes [11], intestinal epithelial cells [12], dendritic cells [13], and monocytes [14], further strengthen the cytoprotection role of TAK1. 
TNF- $\alpha$ contributes to many physiological and pathological processes and plays an important role in mediating survival signaling, apoptosis and necroptosis depending on the cell types and cellular context [1]. On TNF- $\alpha$ binding, TNF receptor 1 (TNFR1) undergoes a conformational change to form TNFR complex I containing TRADD, receptor-interacting protein (RIP)1, cIAPs, TRAF2 and TRAF5. RIP1 ubiquitylation by cIAP1 or TRAF2 can recruit the TAK1 to initiate the canonical NF- $\mathrm{B}$ survival pathway $[1,4,10,15]$. Upon inhibition of NF-kB signaling, for example by de-ubiquitylation of RIP1 and loss of cIAPs, TNFR complex II (TRADD, FADD, caspase 8, RIP1 and RIP3) can be formed and execute two distinct types of cell death, apoptosis and necroptosis that compete for each other and is switched by TAK1 and caspases. Normally, caspase 8 triggers apoptosis by cleaving RIP1 to inhibit the pro-survival actions derived from NF- $\mathrm{BB}$ signaling, and the pro-apoptotic protein Bid to generate a truncated form (tBid) for inducing cytochrome c release and apoptosome formation. However, when caspase 8 is blocked by pharmacological or genetic interventions, RIP1 can recruit RIP3 to form the RIP1/RIP3 necrosome. Within this necrosome, RIP1 and RIP3 phosphorylate each other, further stabilizing the complex and engaging the effector mechanisms of necroptosis, which is a recently identified programmed cell death $[16,17]$. Necroptosis has been shown to rely on mitochondrial reactive oxygen species (ROS) production and disintegration of mitochondrial, lysosomal and plasma membranes [18-20]. Moreover, necroptosis has been shown to involve in the pathogenesis of various diseases, such as ischemic injury, neurodegeneration, viral infection, liver injury, traumatic brain injury, and severe drug reaction [21].

Notably, recent studies further demonstrate the role of TAK1 in controlling the RIP1-dependent apoptosis triggered by TNFR complex II. Besides inducing NF$\kappa \mathrm{B}$-dependent anti-apoptotic mechanisms, an NF-кBindependent cell survival pathway downstream of TAK1 and involving the regulation of ROS-cIAPs-RIP1-caspase pathway has been suggested [2, 22, 23]. Moreover, in mouse embryonic fibroblasts (MEF), TAK1 activity was shown to be a switch between apoptosis and necroptosis following TNF- $\alpha$ stimulation [24]. Hyperactivation of TAK1 possibly through inhibition of caspase-1 leads to necroptosis in dermal fibroblasts which results in the delay in wound healing. In contrast, ablation of TAK1 causes RIP1- and caspase-dependent apoptosis in MEF and monocytes $[14,24]$. Since TAK1 is a potential therapeutic target of inflammatory disorders and cancer [25], it is essential to more understand the role of TAK1 in cell death regulation in different cell types. Moreover, since the role of TAK1 in controlling RIP1-dependent apoptosis was just recently raised and demonstrated only in limited cell types under certain cellular context (e.g. under TAK deficiency plus treatment with either TNF- $\alpha$ or Smac mimetic), it is warrant to further elucidate the detailed regulatory mechanisms underlying this event. Therefore, in this study using 5Z-7-oxozeaenol (TAKI), which is a natural product of fungal origin and an irreversible TAK1 inhibitor [26], we found a cell type specific action of TAKI in apoptosis, and this action occurring in myeloid cells is dependent on endogenously constitutive TNFR-mediated RIP1 activation and mitochondrial ROS production.

\section{Methods}

\section{Cell culture}

Bone marrow-derived macrophages (BMDM) were isolated from 8-12 week old C57BL/6 mice as we previously described [27]. Lung cancer CL1.0 cell line was provided by Dr. Zhixin Yang (National Taiwan University, Taipei, Taiwan). Murine RAW264.7 macrophages, and other cell lines were obtained from the American Type Culture Collection (Manassas, VA, USA). The normal human epidermal keratinocytes (NHEKs) were prepared as we previously described according to the Mackay Memorial Hospital Institutional Review Board (IRB 12MMHIS193) [28]. The CL1.0, HeLa, BV-2, RAW264.7, J774, A431 and CHME3 cells were cultured in Dulbecco's modified Eagle's medium (DMEM). The HCT116 and THP-1 cells were cultured in RPMI 1640 medium. The human retinal pigment epithelial cells (ARPE) were cultured in a 1:1 mixture of DMEM and Ham's F12 medium. Human dermal microvascular endothelial cells (DMVEC) were cultured in Vascular Cell Basal Medium containing Kit-VEGF (ATCC ${ }^{\bullet}$ PCS100 041 $1^{\mathrm{TM}}$ ). NHEKs were cultured in Keratinocyte-SFM (Gibco BRL/Invitrogen, Carlsbad, CA) supplemented with recombinant epidermal growth factor $(0.1-0.2 \mathrm{ng} / \mathrm{ml})$ and bovine pituitary extract $(20-30 \mathrm{mg} / \mathrm{ml})$. All culture media contain $10 \%$ (v/v) heat-inactivated FBS, $100 \mathrm{U} / \mathrm{ml}$ penicillin and $100 \mu \mathrm{g} / \mathrm{ml}$ streptomycin. Cells were incubated at $37{ }^{\circ} \mathrm{C}$ in a humidified atmosphere of $5 \% \mathrm{CO}_{2}$ in air.

Measurement of cell viability by MTT assay and LDH release After indicated drug treatment, cells $\left(2 \times 10^{4} / \mathrm{ml}\right)$ in 96 well plates were incubated with $1 \mathrm{mg} / \mathrm{ml} \mathrm{3-(4,5-dimethyl-}$ thiazol-2-yl)-2,5-diphenyltetrazolium bromide (MTT) at $37^{\circ} \mathrm{C}$ for $60 \mathrm{~min}$. The culture medium was removed, and then the formazan granules generated by live cells were dissolved in DMSO. The net absorbance $\left(\mathrm{OD}_{550}-\mathrm{OD}_{630}\right)$ indicating the enzymatic activity of mitochondria and cell viability was measured. In some experiments, cytotoxicity was assessed by measuring the release from damaged cells of the cytosolic enzyme lactic dehydrogenase (LDH). After treating cells with the indicated agents, LDH activity present in the culture medium was determined using a LDH diagnostic kit (Promega, Heidelberg, Germany). The absorbance values were read at $\mathrm{OD}_{490}$ on a Synergy HT 
Multi-Detection Reader (Bio-Tek Instruments, Winooski, VT, USA). Data were expressed as the percentages of total cellular amount of LDH that was determined by lysing cells with lysis buffer (0.9\% Triton X-100).

\section{Intracellular ATP assay}

Intracellular ATP was determined by CellTiter-Glo ${ }^{\circ} \mathrm{Lu}-$ minescent Kit (G7571, Promega Heidelberg, Germany) according to the manufacturer's protocol. After indicated drug treatment, medium was replaced with the new one, and the reagent in the kit was added to lyse the cells. The plate was gently shaken for 2 min, placed stationary in the dark for $10 \mathrm{~min}$, and then $150 \mu \mathrm{l}$ solution was transferred to a 96-well plate for luminescence detection using LB96V MicroLumat plus (American Laboratory Trading, Boston, USA). With background subtraction, the values were normalized to individual control group as $100 \%$.

\section{Intracellular ROS detection}

After being treated for the indicated time periods, cells were collected and washed with PBS twice, then incubated in PBS containing $5 \mu \mathrm{M}$ DCFH-DA (for detecting the cytosolic ROS) or MitoSOX red (for detecting superoxide in the mitochondria of live cells) for $30 \mathrm{~min}$ at $37^{\circ} \mathrm{C}$. After incubation, cells were immediately subjected to flow-cytometry analysis using the FACScan. The data were analyzed with the CellQuest program.

\section{Mitochondrial respiration measurement}

The oxygen consumption rate (OCR) was measured by the Extracellular flux analyzer XF24 (Seahorse Bioscience, Houston, TX, USA). About 50,000 BMDM cells were plated on laminin-coated XF24 plates and cultured for $24 \mathrm{~h}$ in a $5 \% \mathrm{CO}_{2}$ incubator at $37{ }^{\circ} \mathrm{C}$. Then, the medium was removed and replaced by $500 \mu \mathrm{l} \mathrm{FX}$ assay medium (143 mM NaCl, $5.4 \mathrm{mM} \mathrm{KCl,} 0.8 \mathrm{mM} \mathrm{MgSO}_{4}$, $1.8 \mathrm{mM} \mathrm{CaCl} 2,0.91 \mathrm{mM} \mathrm{Na}_{2} \mathrm{HPO}_{4}, 2 \mathrm{mM}$ glutamine, $2 \mathrm{mg} / \mathrm{ml} \mathrm{BSA}, 3 \mathrm{mg} / \mathrm{l}$ phenol red, and other constituents, $\mathrm{pH}$ 7.4.) The BMDM cells were preincubated for $1 \mathrm{~h}$ at $37{ }^{\circ} \mathrm{C}$ in normal atmosphere. Stock solutions $(\times 10)$ of $1 \mu \mathrm{g} / \mathrm{ml}$ LPS, $1 \mu \mathrm{M}$ TAKI or LPS plus TAKI (injection on port A), $10 \mu \mathrm{g} / \mathrm{ml}$ oligomycin (ATP synthase inhibitor in port B), $2.5 \mu \mathrm{M}$ carbonyl cyanide-ptrifluoro methoxyphenylhydrazone (FCCP, a mitochondrial uncoupler of oxidative phosphorylation in port C), and $2.5 \mu \mathrm{M}$ rotenone (an inhibitor of mitochondrial Complex I in port D) were prepared in FX24 assay media and loaded into injection ports respectively, then measurements were obtained at $37^{\circ} \mathrm{C}$.

\section{Statistical evaluation}

Values were expressed as the mean \pm S.E.M. of at least three independent experiments, which were performed in duplicate. Analysis of variance was used to assess the statistical significance of the differences, and $P$ value $<0.05$ was considered statistically significant.

\section{Ethical approval}

The animal experiments were conducted in accordance with institute regulations after receiving approval from the Ethics Committee of the National Taiwan University College of Medicine (No. 20130391).

\section{Results}

\section{TAKI induced apoptotic cell death in BMDM}

Using MTT assay as the index of cell viability, we found that TAKI $(100 \mathrm{nM})$ treatment for $4 \mathrm{~h}$ can induce cell death of BMDM. Since LPS is a potent TAK1 and NF- $\mathrm{KB}$ activator in BMDM, we interested to understand whether TAKI-induced cytotoxicity is affected under LPS treatment. Notably we found that LPS at sub-cytotoxic concentration $(100 \mathrm{ng} / \mathrm{ml})$ can enhance cell death of TAKI (Fig. 1a). Measuring intracellular ATP level also showed the cytotoxic effect of TAKI and lower ATP content under simultaneous activation of TLR4 (Fig. 1b). To verify the cell death mode, we determined Annexin V and PI staining. Results showed that cell population of Annexin V positive staining, either with or without higher PI staining, was increased along with the time of TAKI treatment (Fig. 1c). In agreement with data of MTT and ATP assays, TAKI-induced increase of Annexin V-positive cell number was elevated in the presence of LPS (Fig. 1c).

Next using PI uptake for necrosis assessment, we found the amount of cells with positive PI staining was increased by TAKI, and was also enhanced in the presence of LPS (Fig. 1d). Further determining the cell necrotic LDH release, we found the concentration- (Fig. 1e) and time(Fig. 1f) dependent effects of LPS to enhance the TAKIinduced response. Using TLR4 ${ }^{-1-}$ BMDM, we confirmed the potentiation effect of LPS on TAKI-induced cell death is resulting from TLR4 activation (Fig. 1g). Given that PI uptake and LDH release were induced along with the increased Annexin V staining, it is suggested that the cell death elicited by TAKI is apoptosis followed by the fast proceeding to necrosis.

\section{TAKI-induced apoptosis depends on RIP1}

Confirming apoptotic feature, TAKI can induce active caspase 8 and caspase 3 formation, and PARP1 cleavage (Fig. 2a). Although LPS co-treatment facilitated the downregulation of pro-caspase 3 and PARP1, it was hard to detect the increased cleavage forms of both proteins. We speculate this is possibly due to the instability of both cleaved proteins. These results suggest that LPS stimulation can enhance TAKI-elicited apoptotic caspase activation. Since TAK1 has been shown to regulate autophagy in breast epithelial cells MCF10A and human cervical 


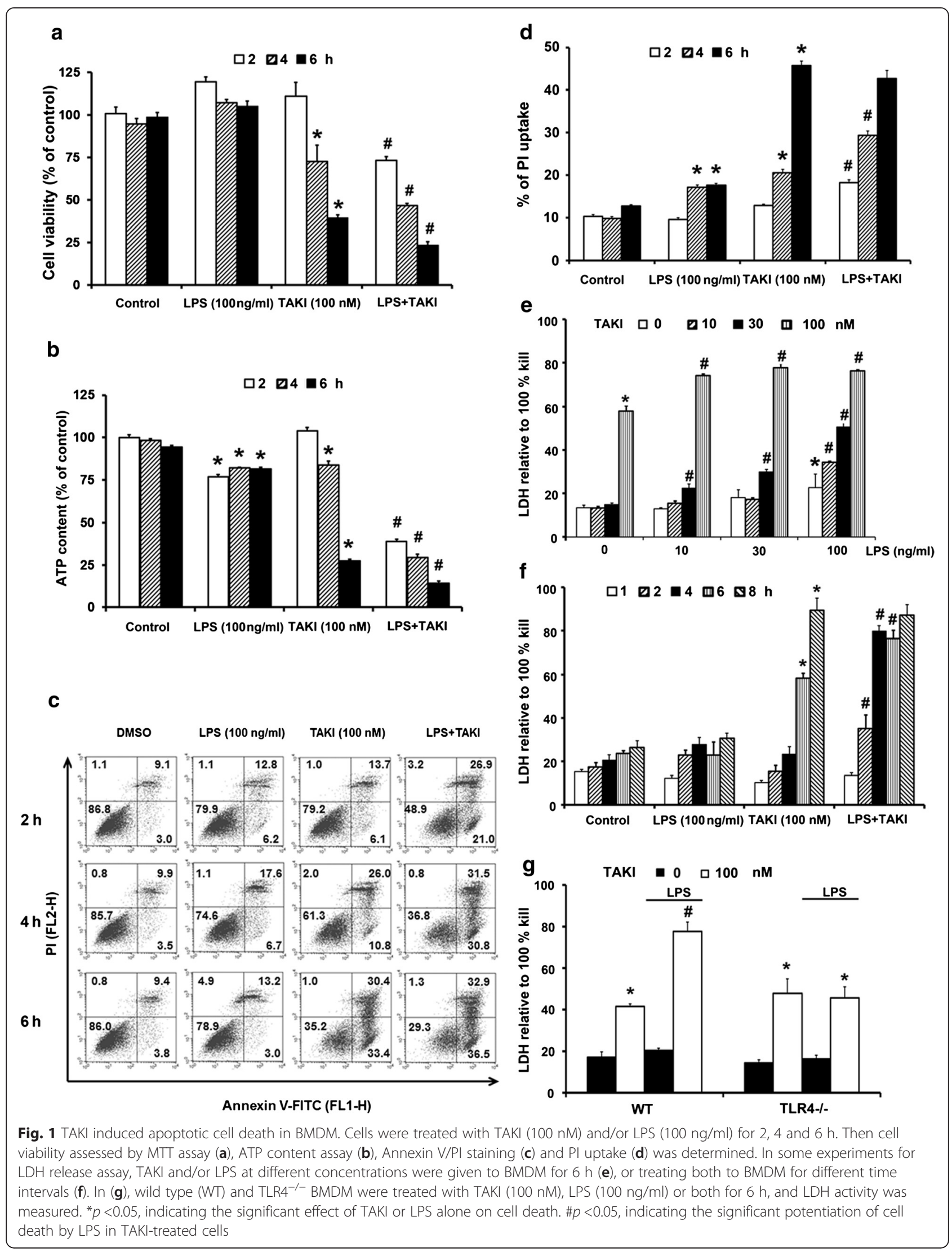




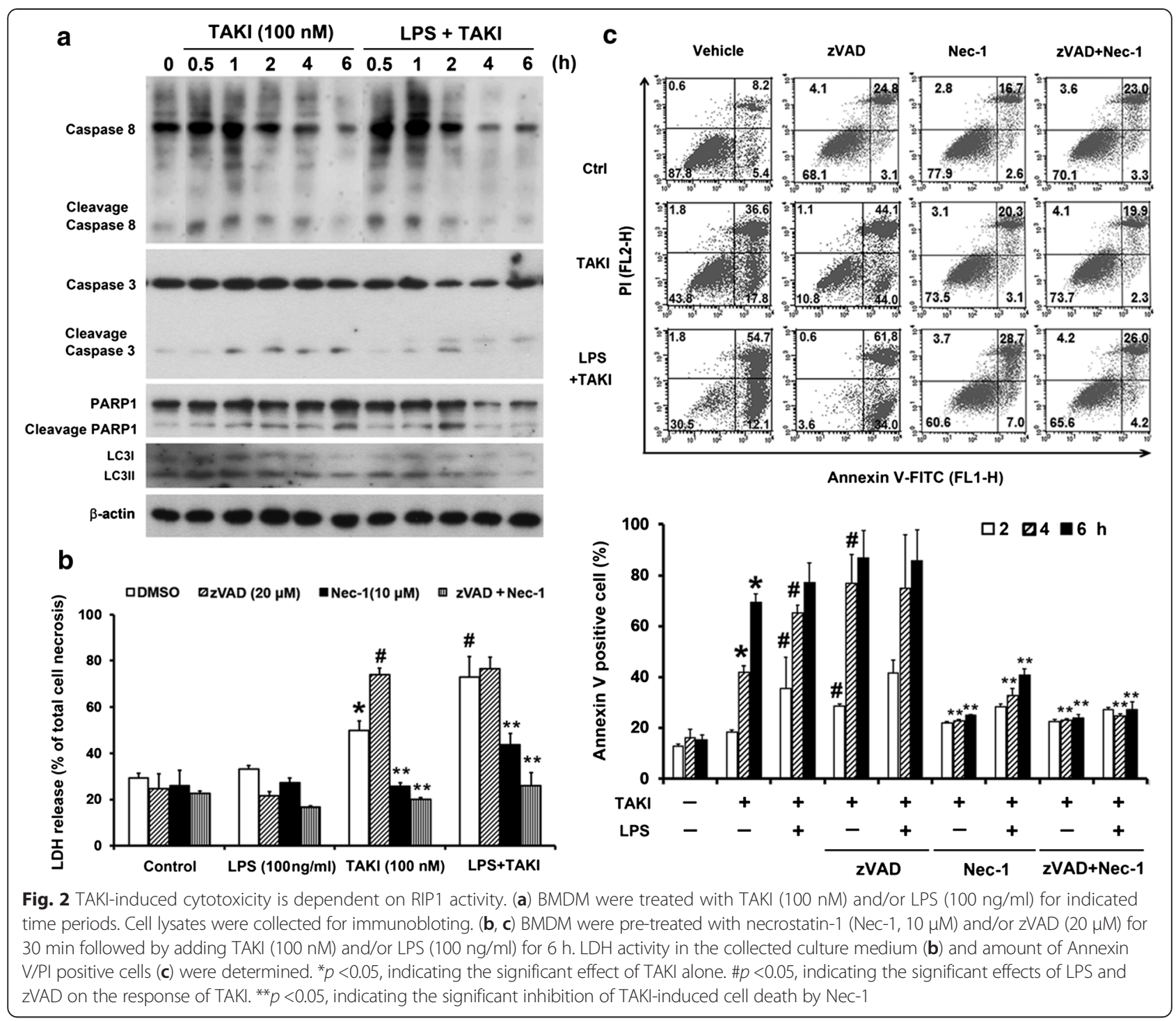

carcinoma HeLa cells $[29,30]$, we wondered whether this event occurs in BMDM. When autophagy is activated, LC3II [LC3-phosphatidylethanolamine (PE)] formation is prerequisite for autophagosome formation and is regarded as an autophagy marker [31]. Results of immunobloting showed no increased effect of TAKI, with or without LPS co-treatment, on LC3II/LC3I ratio (Fig. 2a).

To characterize the cell death pathway in BMDM, we examined the role of RIP1. To this end, we pre-treated cells with $10 \mu \mathrm{M}$ necrostatin-1 (Nec-1, a selective RIP1 inhibitor) and found that $\mathrm{Nec}-1$ can protect cells from TAKI-induced LDH release (Fig. 2b) and Annexin V staining (Fig. 2c). Such protection was also observed in cells with LPS and TAKI co-treatment. Since non-selective caspase inhibitor zVAD was reported to sensitize macrophages and MEF for RIP1-dependent necroptosis [32-34], we further treated macrophages with zVAD to clarify the cell death effects of TAKI. Results revealed that zVAD itself did not affect cell viability (Fig. 2b, c), but its presence further potentiated the TAKI-induced cytotoxicity. Nevertheless when enhanced cytotoxicity was already induced by TAKI + LPS, zVAD no longer increased the cell toxicity. Moreover, the death induced by TAKI + LPS, no matter in the presence or not of zVAD, was diminished by Nec-1 (Fig. 2b, c). These results suggest the involvement of RIP1 in TAKI-induced cell death regardless of the presence of LPS or zVAD.

\section{TAKI-induced cytotoxicity depends on ROS}

Since ROS play an important role in apoptosis and necrosis, we used DCFH-DA and MitoSOX staining to determine cytosolic and mitochondrial ROS levels respectively. The data revealed that both TAKI (100 nM) and LPS (100 ng/ml) can increase cytosolic ROS level (Fig. 3a) with similar extent, and their co-treatment was non-additive (Fig. 3a). Compared to the early onset (30 min) of ROS 


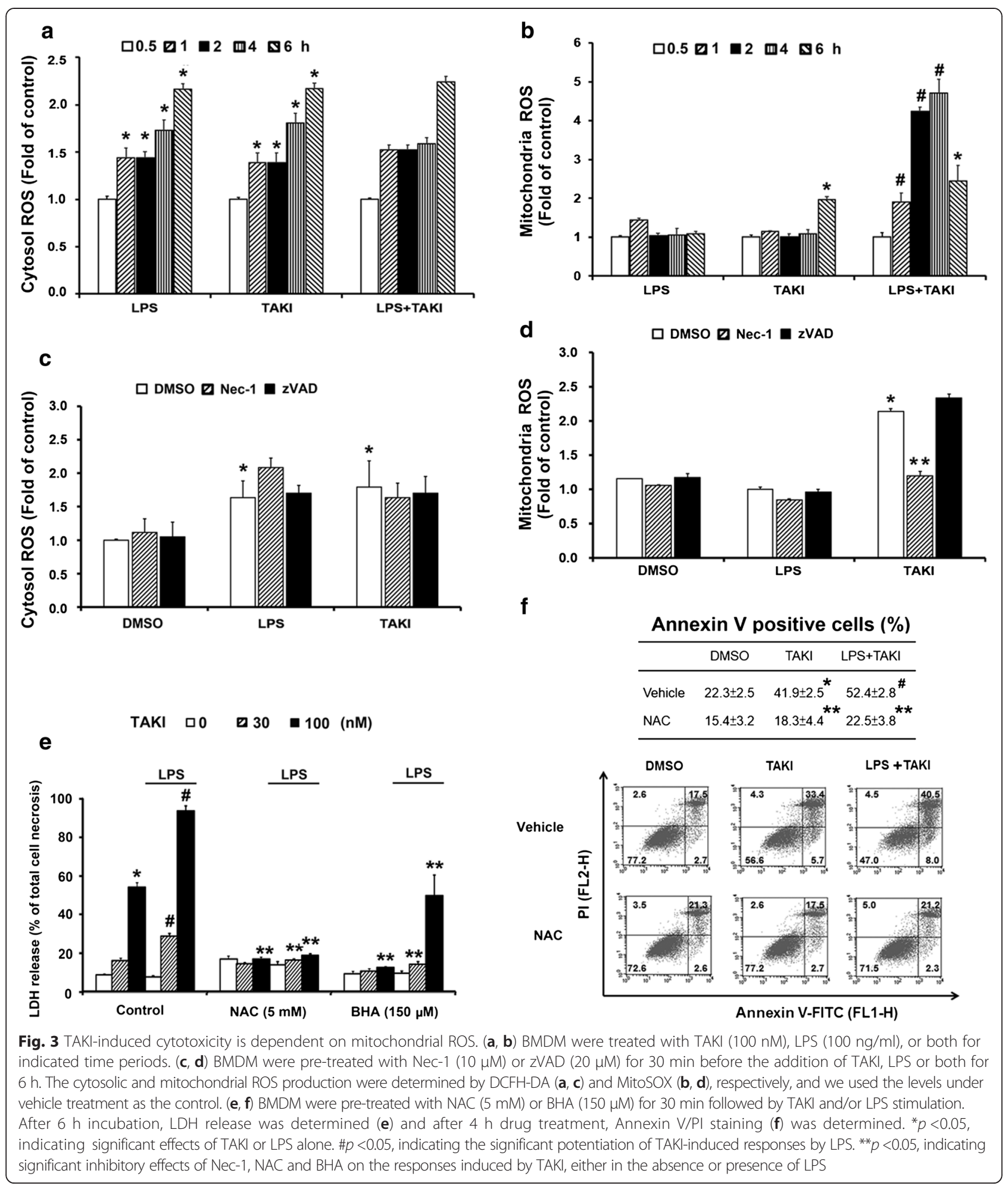

production in cytosol, mitochondrial ROS production was observed at $6 \mathrm{~h}$ after TAKI treatment. Although LPS alone did not cause significant mitochondrial ROS production, its co-treatment with TAKI led to an enhancement of mitochondrial ROS production in terms of its onset and extent (Fig. 3b). Due to the apparent cytotoxicity, we did not detect a sustained mitochondrial ROS increase at $6 \mathrm{~h}$ in TAKI + LPS group. Accordingly we tested the effects of Nec-1, NAC (a general antioxidant through production of intracellular GSH), BHA (a ROS scavenger) and zVAD on 
ROS response. Since the onset of TAKI-induced cytosolic ROS production is much earlier than that of mitochondrial ROS, and RIP1-mediated mitochondrial ROS is implicated in necroptotic cell death [35], we wonder if the early increased cytosolic ROS is also mediated by RIP1. As a result, Nec-1 can reduce the mitochondrial but not cytosolic ROS increase induced by TAKI (Fig. 3c, d). On the other hand, zVAD did not cause any changes in this aspect (Fig. 3c, d). In addition, NAC and BHA can markedly reduce $\mathrm{LDH}$ release induced by TAKI/LPS (Fig. 3e), and NAC also reduced the amount of Annexin V positive cells in response to TAKI (Fig. 3f). These results suggest the prerequisite role of mitochondrial ROS production triggered by RIP1 rather than cytosolic ROS in TAKI-induced cell death.

\section{No contribution of IKK and MAPKs to TAKI-induced cell death}

Apart from RIP1, we wonder the roles of MAPKs and IKK in TAKI-induced cell death. Immunobloting data revealed the abilities of TAKI to inhibit TAK1, IKK, ERK, p38 and JNK activation induced by LPS (Fig. 4a). Since TAKI can inhibit but not abolish IKK and MAPKs activation under LPS stimulation, we wondered if these kinases are involved to regulate cell death mechanisms under TAKI and/or LPS stimulation. Then we tested the effects of BMS345541, SB203580, SP600125, and U0126, inhibitors of IKK, p38, JNK and ERK, respectively, on TAKI-induced cell death. As shown in Fig. 4b, BMS345541 treatment alone can induce moderate cell death and this effect was further enhanced by LPS and TAKI. In contrast, MAPKs inhibitors failed to affect the death induced by TAKI, either in the presence or absence of LPS (Fig. 4b).
TAKI-induced cell death involves mitochondria dysfunction Since apoptosis is characterized as a consequence of mitochondrial dysfunction, we interested to understand if TAKI afffects the functions of mitochondria. First, we found that TAKI treatment caused the loss of mitochondrial membrane potential, which was unchanged by LPS, but was reversed by Nec-1 (Fig. 5a). Notably as the increased cell death response shown above, zVAD augmented mitochondrial membrane potential loss caused by TAKI/LPS (Fig. 5a). Next, we analyzed mitochondrial oxygen consumption rate (OCR) which reflects overall metabolic activity of mitochondria. As shown in Fig. 5b, TAKI treatment can gradually decrease OCR, while LPS itself did not induce significant OCR change. Nevertheless LPS co-treatment can accelerate the effect of TAKI. After drug treatment for $2 \mathrm{~h}$, we added standard agents oligomycin, FCCP and rotenone to determine the mitochondrial respiration functions. We found oligomycin can rapidly decrease OCR levels in control and LPS groups, but not in TAKI and TAKI/LPS groups when their OCR level was markedly decreased. Meanwhile, unlike the general effects of FCCP and rotenone in control and LPS groups, both mitochondrial tested agents also cannot alter OCR in cells treated with TAKI, either with or without LPS (Fig. 5b).

\section{Intrinsic action of TNF-a contributes to TAKI-induced cell death}

Since TNF- $\alpha$ has been shown to induce cell death of various features depending on the cellular context, we wondered its involvement in TAKI-treated macrophages. Using neutralizing TNF- $\alpha$ antibody and enbrel (soluble TNF- $\alpha$ receptor), we found that the cell death induced by TAKI,

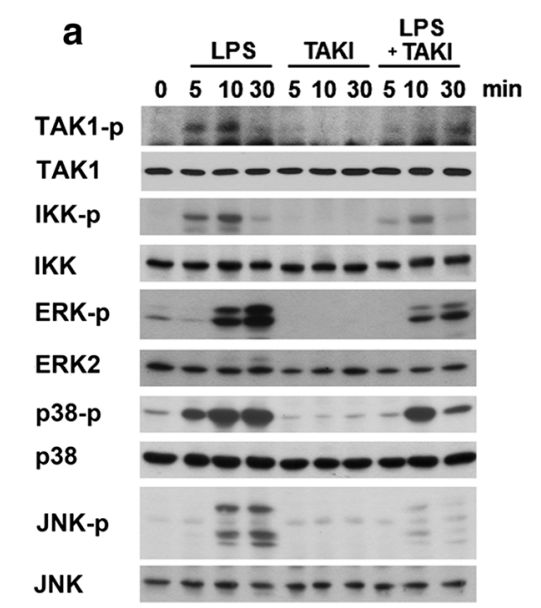

\section{b}

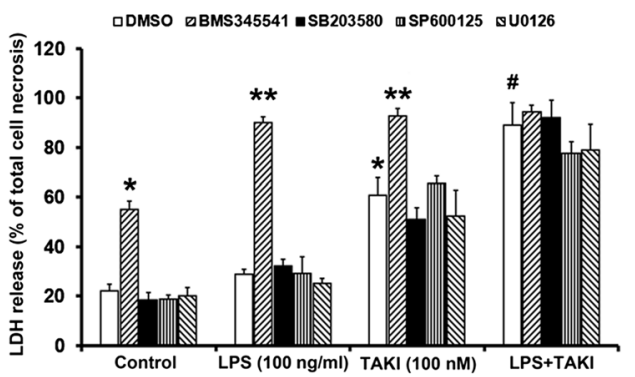

Fig. 4 TAKI inhibits LPS-induced IKK, JNK, p38 and ERK activation. (a) BMDM were treated with LPS (100 ng/ml), TAKI (100 nM) or both for indicated time intervials, and then immunobloting against specific proteins was conducted. (b) BMDM were pretreated with BMS345541 (3 $\mu$ M), SB230580

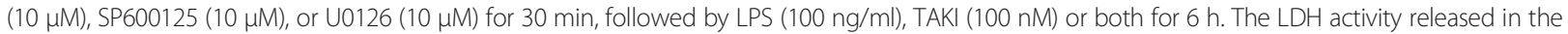
culture medium was then determined. ${ }^{*} p<0.05$, indicating significant effects of TAKI alone. \#p $<0.05$, indicating the significant potentiation of TAKI-induced responses by LPS. ${ }^{* *} \mathrm{p}<0.5$, indicating significant potentiation of TAKI or LPS response by BMS345541 


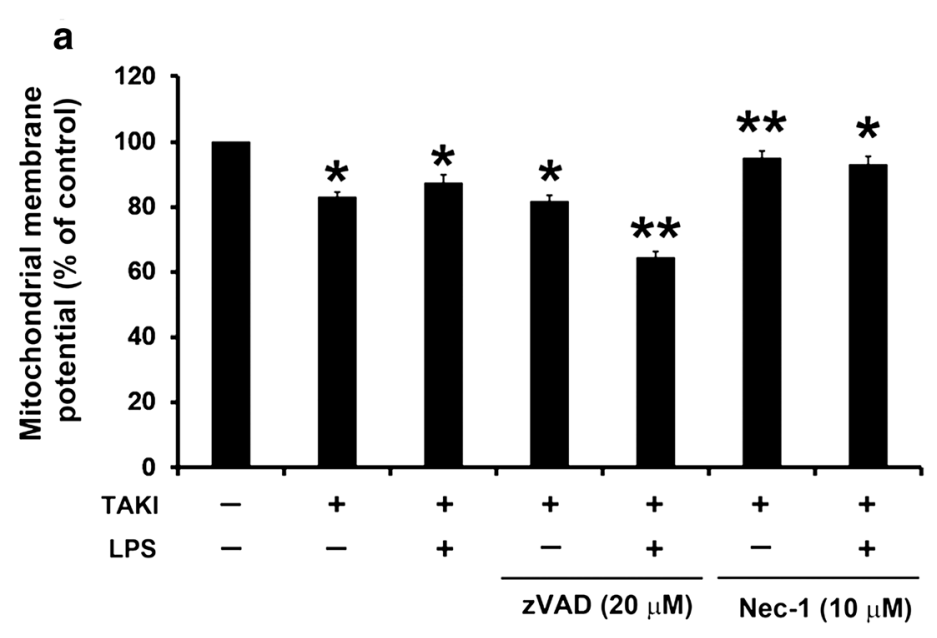

b

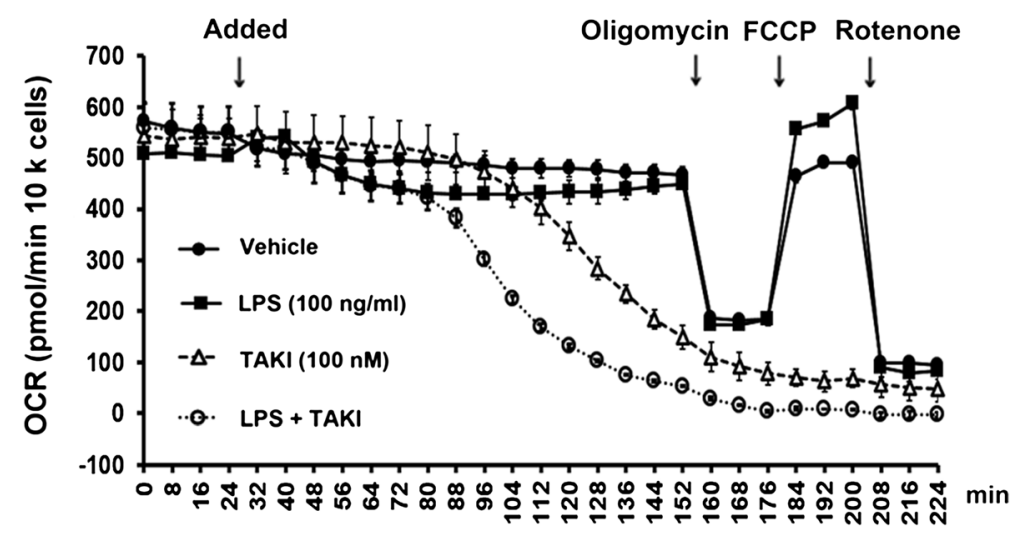

Fig. 5 TAKI-induced cell death involves mitochondrial dysfunction. (a) BMDM were treated with $20 \mu M$ z VAD or $10 \mu M$ Nec-1 for 30 min, followed by TAKI (100 nM) and/or LPS (100 ng/ml) for $6 \mathrm{~h}$. Then mitochondria membrane potential was detected. * $p<0.05$, indicating significant reduction of mitochondrial membrane potential caused by TAKI. ${ }^{* *} p<0.05$, indicating significant potentiation or inhibiion of mitochondrial membrane potential loss by zVAD and Nec-1, respectively. (b) The mitochondrial metabolism was assessed by using the seahorse XF24 to measure the oxygen consumption rate (OCR). BMDM were treated with LPS (100 ng/ml), TAKI (100 nM), or LPS plus TAKI at 32 min as indicated with arrow. The inhibitor of ATP synthase, oligomycin $(10 \mu \mathrm{g} / \mathrm{ml})$ was added to discriminate ATP-linked respiration and proton leak. After adding an uncoupler of mitochondrial oxidative phosphorylation, FCCP $(2.5 \mu \mathrm{M})$, OCR was raised to the maximal respiratory capacity. Finally rotenone (2.5 $\mu \mathrm{M})$ was added to block the electron transport chain of oxidative phosphorylation

regardless of the presence of LPS or not, was inhibited by both treatments (Fig. 6a). Accordingly enbrel can reduce cytosolic and mitochondrial ROS production caused by TAKI alone (Fig. 6b, c), and LPS-induced cytosolic ROS increase was also diminished by enbrel (Fig. 6b). These findings suggest that autocrine or paracrine action of TNF$\alpha$ is involved in TAKI-induced ROS production and subsequent cytotoxicity. Next, the TNF- $\alpha$ production was determined by ELISA in order to correlate with cell death response. Surprisingly although TNF- $\alpha$ antibody and enbrel can abrogate TAKI-induced cell death and ROS production, no significant increase of TNF- $\alpha$ release under TAKI treatment was found. Stimulating macrophages with LPS as expected increased TNF- $\alpha$ production, and this effect was abrogated by the co-treatment with TAKI (Fig. 6d).
Besides TNF- $\alpha$, we also wondered the role of type I IFNs in cell death, as IFNs have been shown to regulate cell death in macrophages [36]. As shown in Fig. 6a, antagonistic IFN antibody had minimal effect on TAKI-induced cell death. These results all together suggest that the intrinsic and constitutive action of TNF- $\alpha$ is prerequisite for TAKIinduced ROS production and the subsequent cell death.

TAKI-induced cell death exhibits the cell type specificity Apart from BMDM, we also tested the effects of TAKI in other murine macrophages (RAW264.7 and J774) and microglial (BV-2) cell lines. Using necrotic marker LDH release as index, we found TAKI treatment also significantly induces cell death in these cell types with BV-2 displaying the prominent extent (Fig. 7a). Consistently $\mathrm{Nec}-1$ and 


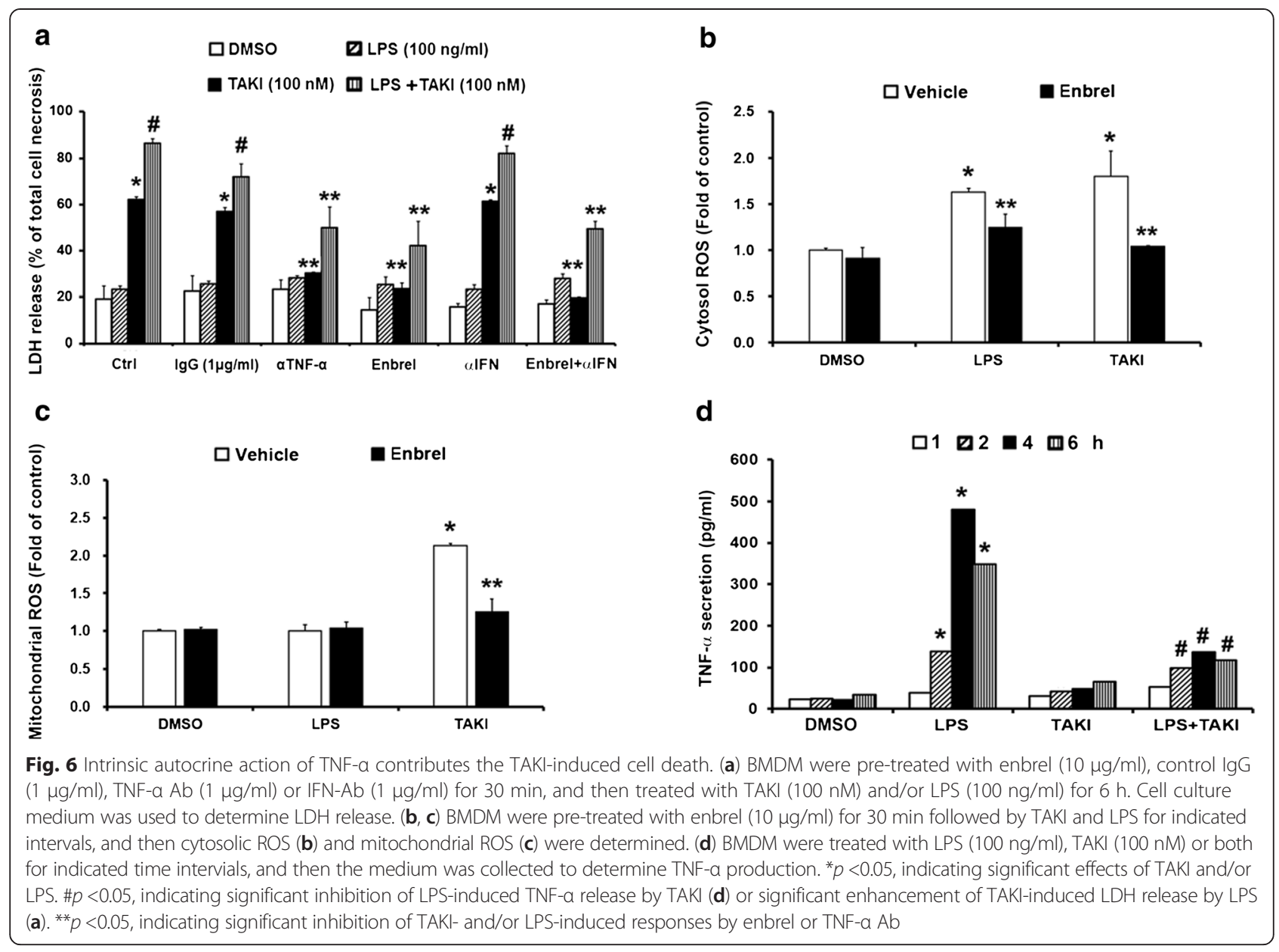

enbrel exerted the inhibitory effects on TAKI-induced cell death in terms of MTT (Fig. 7b) and ATP assays (Fig. 7c). Notably zVAD treatment can reverse the cytotoxicity of TAKI in BV-2 cells, but not in RAW264.7 cells. In order to understand the cytotoxicity effect of TAKI is general or not, we examined several cell types. We found that TAKI at concentrations up to $100 \mathrm{nM}$ failed to alter the cell viability in human THP-1 monocytes, THP-1-derived macrophages, and CHME, 3 microglia. Neither normal human epidermal keratinocyte (NHEK), retinal pigment epithelial cells (ARPE), dermal microvascular endothelial cells (DMVEC) or cancer cells like A431 squamous cell carcinoma, CL1.0 lung cancer, HeLa cervical cancer, and HCT116 colon cancer was affected by TAKI (Fig. 7a, d, e). To clear if less intrinsic TNF- $\alpha$ action made such susceptibility difference, we co-treated TNF- $\alpha$ (10 ng/ml) together with TAKI in these non-responsive cell types. As a result, TAKI still cannot induce cytotoxicity upon TNF- $\alpha$ cotreatment by either MTT (Fig. 7d) or ATP assays (Fig. 7e). After observing this, we wondered if distinct expression level of endogenous TNFR determines the susceptibility in response to TAKI + TNF- $\alpha$. We thus determined the basal level of TNFR in these cell types. Immunobloting data revealed that BV-2, HCT116 and NHEK expressed higher and comparatively equivalent level of TNFR1, RAW264.7, A431 and ARPE expressed moderate amount, and BMDM expressed the lowest amount (Fig. 7f). Since TNFR1 expression level does not correlate to the susceptibility of TNF- $\alpha$-dependent cytotoxicity, we suggest it is the cellular context rather than TNF- $\alpha$ signaling determines the cell viability in response to TAKI.

\section{Discussion}

Given that TAK1 exerts multifaceted functions in controlling cell fate and inflammatory responses, targeting TAK1 for inflammatory disorders and cancer has raised great attention. The therapeutic potential of TAKI in enhanced chemotherapeutic efficacy might result from its potent inhibition of the IKK-NF- $\mathrm{KB}$ pathway, potentiation of ROS and caspase activity [37-39]. In this aspect, previous studies demonstrated the effectiveness of TAK1 inhibition or deficiency in skin cancer [40], multiple lymphoma [41], KRAS-dependent colon cancers [42], breast cancer [37] and cervical cancer [38]. Moreover, a TAKI closely analog, E6201 is currently in phase II clinical trials for the treatment of melanoma [43], and may be useful for 


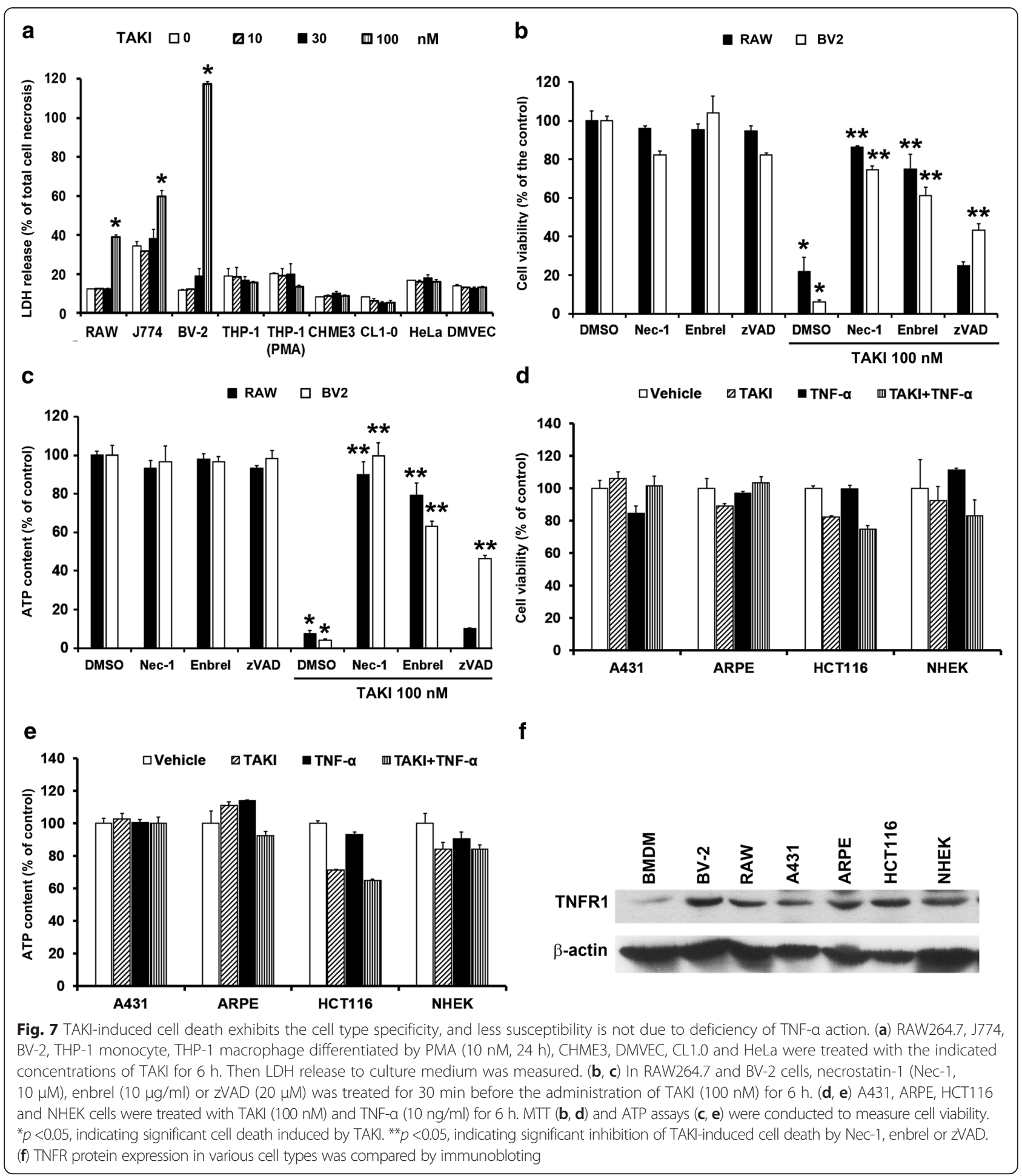

psoriasis [44] and arthritis [45]. On the other hand, the neuroprotective effects of TAK inhibition for stroke, traumatic brain injury and neurodegenerative diseases are associated with a reduced activation of JNK that leads to inflammation and apoptosis [46-48].
According to its multifaceted signaling functions as mentioned above, TAK regulation of cell viability is believed to be cell type and cell context dependent. Since macrophages play a major role in acute inflammatory responses in various tissues and diseases [49], and TAK1 
knockout leads to the automatic macrophage death $[2,50,51]$, in this study we used macrophages as the cell model to deep in the death regulating mechanisms and signaling pathways controlled by TAK1. Our present data showed that TAKI can induce a dramatic cell death in primary BMDM. Different from previous finding showing the delayed onset of spontaneous cell death in $\mathrm{TAK}^{-1-}$ BMDM (around $45 \%$ cell death at 4 day) [50]. TAKIinduced cell death in BMDM is of rapid onset (around $60 \%$ cell death at $6 \mathrm{~h}$ treatment). Currently we do not have clear explanation for this difference, but effects of TAK1 gene deletion on in vitro macrophage differentiation and/ or some cellular adaptive regulation cannot be excluded. In our study, we found the death caused by TAKI in BMDM is of caspase-dependent apoptosis mode. The supporting evidence of this notion includes the appearance of apoptotic landmark Annexin V (Fig. 1c), and activation of caspases 8 and 3 (Fig. 2a). Here we rule out the involvement of LC3 dependent autophagy or PARP1 dependent cell death mode (i.e. parthanatos) in this action, because TAKI cannot trigger LC3II increase (Fig. 2a) and its induced cell death is not affected by PARP1 inhibitor (data not shown). So our result is in agreement with previous report showing apoptosis occurring in $\mathrm{TAK}^{-/-}$BMDM [50]. A study reported by Morioka et al. also showed that ablation of TAK1 in MEF led to caspase-dependent apoptosis [24].

This study for the first time shows the ability of TAK inhibitor alone to induce RIP1-dependent apoptosis, unlike the case requiring exogenous TNF- $\alpha$ to trigger RIP1-dependent apoptosis in MEF with TAK1-deficient or inhibition condition [52]. Another interesting finding of our study is that constitutive TNFR signal through autocrine loop plays an important role to control the death action of TAKI in BMDM, RAW264.7 macrophages and BV-2 microglia. Although TAKI alone is not able to change basal TNF- $\alpha$ production (Fig. $6 \mathrm{~d}$ ), TAKI-induced ROS production (Fig. 6b, c) and cell death (Fig. 6a) were abrogated by enbrel and neutralizing antibody of TNF- $\alpha$. These findings indicate the crucial determinant of autocrine factor TNF- $\alpha$ to timely control the cell fate in macrophages and microglia. Even lower amount of TNF- $\alpha$ secreted at resting state is sufficient to render such death regulation action. Indeed as previously reported, depending on the statuses of caspase, RIP1 and TAK1 activity, exogenous TNF- $\alpha$ can mediate cell survival or various modes of cell death [2]. Thus our current findings support the complexity of TNFR signaling pathways in coordination with TAK1 activity to timely control the cell fate. Moreover, we for the first time to strengthen the key role of constitutive and autocrine action of TNF- $\alpha$ in controlling macrophages and microglia survival. Such intrinsic and quiescent function of TNF- $\alpha$ can be unmasked and become sensitized to impact cellular response when TAK1-dependent protection signals and functions are severely impaired.
In this study using TAK inhibitor we demonstrated the cell type specificity for TAKI-induced cytotoxicity. We found TAKI itself can induce cell death in murine macrophages (BMDM, RAW264.7, J774) and microglia (BV-2), but not in cancer cells (CL1.0, HeLa, HCT116, A431), human THP-1 monocytes, human CHME3 microglia, and primary normal cells (NHEK, ARPE and DMVEC) (Fig. 7). These data strongly suggest that TAK-dependent cell signaling and molecular events in regulating cell viability are cell type specific. In addition, although TNFR signaling as discussed above is important to mediate TAKI-induced cell death in BMDM, RAW264.7 and BV-2, it is not the major factor to explain why there is no death response to TAKI in other cell types. This is because constitutive TNFR1 level in various cell types does not correlate to the cell death susceptibility in response to TAKI plus TNF- $\alpha$ (Fig. 7a, e, f). Although TNFR1 rather than TNFR2 is known as the major receptor for TNF- $\alpha$-induced signaling and cellular functions, our current study still cannot exclude the potential role of TNFR2 in controlling the cell viability of macrophages and microglia in response to TAKI. Therefore, it is the distinct cellular context rather than TNF- $\alpha$ signals via TNFR1 alone to determine the cell susceptibility to TAKI.

Previous studies conducting in TAK1 deletion cell models have demonstrated the role of ROS accumulation for cell death $[53,54]$. In our study using TAKI we similarly observed the increased cytosolic and mitochondrial ROS production from TNFR signals in BMDM (Fig. 6b, c). Moreover, RIP1 inhibition by necrostatin-1 only reduces mitochondrial but not cytosolic ROS elevation (Fig. 3c, d). TNFR1 activation has been shown to activate NADPH oxidase for cytosolic ROS production, which causes cIAP protein depletion and activation of RIP1-dependent caspase-8 [2]. Besides activating caspase-8, RIP1 activation also leads to ROS production in mitochondria [52]. Based on these findings we suggest that TAK inhibition can unmask intrinsic TNFR signal for the rapid initiation of cytosolic ROS production, which in turn triggers RIP1 activation and late mitochondrial ROS production. Accordingly we suggest the enhanced cell death upon LPS co-treatment is through increased RIP1-dependent mitochondrial ROS production (Fig. 3b). Previously TLR4 activation by LPS was shown to activate RIP1 through TRIF signaling pathway [55]. Similar to TNF- $\alpha$, NO has been shown to induce programmed cell death in endothelial cells, and both RIP1 and ROS are involved [56]. Herein, we explored the possible role of NO in TAKI-elicited cell death in BMDM. We found that treatment with iNOS inhibitor L-NAME did not affect cell death induced by TAKI, either in the presence or absence of LPS (data not show). In addition, our data suggest the death mechanism caused by TAKI is independent of IKK, because we observed the additive cytotoxicity between TAKI and IKK inhibitor (Fig. 4b). 
Depending on the cell types, caspase inhibition has been shown to switch cell death from apoptosis to either autophagic cell death or necroptosis [14, 32-34, 57]. Notably the induction of necroptosis by caspase inhibitor in macrophages and MEF is also RIP1- and TNFR-dependent [14, 32-34]. In our study, zVAD treatment cannot block TAKI-induced cell apoptosis in BMDM and RAW264.7 macrophages (Fig. 7b, c), but an even more increased cell death was observed in BMDM (Fig. 2c). Such cell death under zVAD treatment in BMDM still depends on RIP1 (Fig. 2b, c). Therefore we suggest zVAD co-treatment can drive cells proceeding a RIP1-dependent but caspaseindependent apoptosis. Supporting this suggestion, under caspase inhibition mitochondrial cell death resulting from loss of respiration, but not from cytotoxic protein release has been reported [58]. Therefore it is still necessary to explore the death mechanism in details under TAKI + zVAD treatment. It is also needed to elucidate the mechanisms underlying the partial protective effect of zVAD in TAKItreated BV-2 cells, which is unlike the effects seen in BMDM and RAW264.7 cells. Moreover whether autophagy is involved in TAKI + zVAD-induced cell death in BMDM needs further investigation.

Taken together, we for the first time demonstrate TAKIinduced cytotoxicity is cell context specific and depends on the endogenously constitutive TNFR signaling. An IKK-independent cell survival pathway downstream of TAK1 is essential for macrophages to prevent apoptosis signal induced by autocrine TNF- $\alpha$ through RIP1-ROScaspase pathway. All these results highlight the complexity of cell death regulation in macrophages in viewing the functional balance of molecules among TNFR, TAKI, RIP1 and caspases. Notably, since TAK inhibition is under drug development for sensitizing chemotherapy in cancer patients and for several inflammation-associated diseases, our current findings are warrant in considering the pros and cons for targeting TAK in disease treatment.

\section{Conclusion}

TAK1 is involved to regulate cell survival. Inhibition of TAK1 leading to cell death is cell context specific. In murine macrophages, TAKI-induced apoptosis is dependent on the constitutive autocrine action of TNF- $\alpha$, RIP1activation and ROS production.

\footnotetext{
Abbreviations

AP-1: Activator protein-1; ARPE: Retinal pigment epithelial cells; BHA: Butylated hydroxyanisol; BMDM: Bone marrow-derived macrophages; DCFH-DA: 2',7'-Dichlorodihydrofluorescein diacetate; DMEM: Dulbecco's modified Eagle's medium; DMVEC: Dermal microvascular endothelial cells; FCCP: Carbonyl cyanide p-(trifluoromethoxy) phenylhydrazon; FMK: Fluoro-methylketone; IL: Interleukin; LDH: Lactic dehydrogenase; LPS: Lipopolysaccharide; MEF: Mouse embryonic fibroblasts; MTT: 3-(4,5-Dimethylthiazol-2-yl)-2,5-diphenyltetrazolium bromide; NAC: N-AcetylL-cysteine; Nec-1: Necrostatin-1; NHEK: Normal human epidermal keratinocytes; OCR: Oxygen consumption rate; PI: Propidium iodide; RIP: Receptor-interacting protein; ROS: Reactive oxygen species; TAK1: Transforming growth factor- $\beta$ (TGF-
}

ß)-activated kinase 1; TAKI: 5Z-7-Oxozeaenol; TLR: Toll-like receptor; TNF: Tumor necrosis factor; TNFR1: TNF receptor 1; zVAD: Benzyloxycarbonyl-Val-Ala-Asp.

\section{Competing interests}

The authors declare that they have no competing interest.

\section{Authors' contributions}

WWL and JSW designed research; JSW and DW performed experiments; JSW, DYH and DW analyzed data; WWL wrote the paper. All authors read and approved the final manuscript.

\section{Acknowledgements}

This work was supported by grants from the National Science Council (NSC 100-2320-B-002-088-MY3), Ministry of Science and Technology (MOST 103-2320-B-002-069-MY3) and Taipei Medical University-Shuang Ho Hospital (103TMU-SHH-06).

\section{Author details}

'Department of Pharmacology, College of Medicine, National Taiwan University, No 1, Sec 1, Jen-Ai Road, Taipei, Taiwan. ${ }^{2}$ Graduate Institute of Medical Sciences, Taipei Medical University, Taipei, Taiwan. ${ }^{3}$ Department of Neurology, Shuang Ho Hospital, Taipei Medical University, New Taipei City, Taiwan.

Received: 26 June 2015 Accepted: 5 September 2015

Published online: 18 September 2015

\section{References}

1. Chen ZJ, Bhoj V, Seth RB. Ubiquitin, TAK1 and IKK: is there a connection? Cell Death Differ. 2006;13:687-92.

2. Mihaly SR, Ninomiya-Tsuji J, Morioka S. TAK1 control of cell death. Cell Death Differ. 2014;21:1667-76.

3. Sato S, Sanjo H, Tsujimura T, Ninomiya-Tsuji J, Yamamoto M, Kawai T, et al. TAK1 is indispensable for development of T cells and prevention of colitis by the generation of regulatory T cells. Int Immunol. 2006;18:1405-11.

4. Shim JH, Xiao C, Paschal AE, Bailey ST, Rao P, Hayden MS, et al. TAK1, but not TAB1 or TAB2, plays an essential role in multiple signaling pathways in vivo. Genes Dev. 2005;19:2668-81.

5. Sorrentino A, Thakur N, Grimsby S, Marcusson A, von Bulow V, Schuster N, et al. The type I TGF-beta receptor engages TRAF6 to activate TAK1 in a receptor kinase-independent manner. Nat Cell Biol. 2008;10:1199-207.

6. Adhikari A, Xu M, Chen ZJ. Ubiquitin-mediated activation of TAK1 and IKK. Oncogene. 2007;26:3214-26.

7. Ninomiya-Tsuji J, Kishimoto K, Hiyama A, Inoue J, Cao Z, Matsumoto K The kinase TAK1 can activate the NIK-I kappaB as well as the MAP kinase cascade in the IL-1 signalling pathway. Nature. 1999;398:252-6.

8. Yamaguchi K, Shirakabe K, Shibuya H, Irie K, Oishi I, Ueno N, et al. Identification of a member of the MAPKKK family as a potential mediator of TGF-beta signal transduction. Science. 1995;270:2008-11.

9. Inokuchi S, Aoyama T, Miura K, Osterreicher CH, Kodama Y, Miyai K, et al. Disruption of TAK1 in hepatocytes causes hepatic injury, inflammation, fibrosis, and carcinogenesis. Proc Natl Acad Sci U S A. 2010;107:844-9.

10. Bettermann K, Vucur M, Haybaeck J, Koppe C, Janssen J, Heymann F, et al. TAK1 suppresses a NEMO-dependent but NF-kappaB-independent pathway to liver cancer. Cancer Cell. 2010;17:481-96.

11. Omori E, Matsumoto K, Sanjo H, Sato S, Akira S, Smart RC, et al. TAK1 is a master regulator of epidermal homeostasis involving skin inflammation and apoptosis. J Biol Chem. 2006;281:19610-7.

12. Kajino-Sakamoto R, Inagaki M, Lippert E, Akira S, Robine S, Matsumoto K, et al. Enterocyte-derived TAK1 signaling prevents epithelium apoptosis and the development of ileitis and colitis. J Immunol. 2008;181:1143-52.

13. Wang Y, Huang G, Vogel P, Neale G, Reizis B, Chi H. Transforming growth factor beta-activated kinase 1 (TAK1)-dependent checkpoint in the survival of dendritic cells promotes immune homeostasis and function. Proc Natl Acad Sci U S A. 2012;109:E343-352.

14. Lamothe B, Lai $Y$, Xie M, Schneider MD, Darnay BG. TAK1 is essential for osteoclast differentiation and is an important modulator of cell death by apoptosis and necroptosis. Mol Cell Biol. 2013;33:582-95.

15. Jadrich $J \mathrm{~L}, \mathrm{O}^{\prime}$ Connor MB, Coucouvanis E. Expression of TAK1, a mediator of TGF-beta and BMP signaling, during mouse embryonic development. Gene Expr Patterns. 2003;3:131-4. 
16. Vanlangenakker N, Vanden BT, Vandenabeele P. Many stimuli pull the necrotic trigger, an overview. Cell Death Differ. 2012;19:75-86.

17. Wu W, Liu P, Li J. Necroptosis: an emerging form of programmed cell death. Crit Rev Oncol Hematol. 2012;82:249-58.

18. Sun L, Wang H, Wang Z, He S, Chen S, Liao D, et al. Mixed lineage kinase domain-like protein mediates necrosis signaling downstream of RIP3 kinase. Cell. 2012;148:213-27.

19. Wang $Z$, Jiang $H$, Chen S, Du F, Wang $X$. The mitochondrial phosphatase PGAM5 functions at the convergence point of multiple necrotic death pathways. Cell. 2012;148:228-43.

20. Zhao J, Jitkaew S, Cai Z, Choksi S, Li Q, Luo J, et al. Mixed lineage kinase domain-like is a key receptor interacting protein 3 downstream component of TNF-induced necrosis. Proc Natl Acad Sci U S A. 2012;109:5322-7.

21. Vandenabeele P, Galluzzi L, Vanden BT, Kroemer G. Molecular mechanisms of necroptosis: an ordered cellular explosion. Nat Rev Mol Cell Biol. 2010;11:700-14.

22. Bertrand MJ, Milutinovic S, Dickson KM, Ho WC, Boudreault A, Durkin J, et al. CIAP1 and CIAP2 facilitate cancer cell survival by functioning as E3 ligases that promote RIP1 ubiquitination. Mol Cell. 2008;30:689-700.

23. Morioka S, Omori E, Kajino T, Kajino-Sakamoto R, Matsumoto K, Ninomiya-Tsuji J. TAK1 kinase determines TRAlL sensitivity by modulating reactive oxygen species and CIAP. Oncogene. 2009:28:2257-65.

24. Morioka S, Broglie P, Omori E, Ikeda Y, Takaesu G, Matsumoto K, et al. TAK1 kinase switches cell fate from apoptosis to necrosis following TNF stimulation. J Cell Biol. 2014;204:607-23.

25. Sakurai H. Targeting of TAK1 in inflammatory disorders and cancer. Trends Pharmacol Sci. 2012;33:522-30.

26. Wu J, Powell F, Larsen NA, Lai Z, Byth KF, Read J, et al. Mechanism and in vitro pharmacology of TAK1 inhibition by (5Z)-7-Oxozeaenol. ACS Chem Biol. 2013:8:643-50.

27. Lin YC, Huang DY, Chu CL, Lin WW. Anti-inflammatory actions of Syk inhibitors in macrophages involve non-specific inhibition of toll-like receptors-mediated JNK signaling pathway. Mol Immunol. 2010;47:1569-78.

28. Wu NL, Lee TA, Tsai TL, Lin WW. TRAlL-induced keratinocyte differentiation requires caspase activation and p63 expression. J Invest Dermatol. 2011;131:874-83.

29. Criollo A, Senovilla L, Authier H, Maiuri MC, Morselli E, Vitale I, et al. The IKK complex contributes to the induction of autophagy. EMBO J. 2010;29:619-31.

30. Herrero-Martin G, Hoyer-Hansen M, Garcia-Garcia C, Fumarola C, Farkas T, Lopez-Rivas A, et al. TAK1 activates AMPK-dependent cytoprotective autophagy in TRAlL-treated epithelial cells. EMBO J. 2009;28:677-85.

31. Reggiori F, Komatsu M, Finley K, Simonsen A. Autophagy: more than a nonselective pathway. Int J Cell Biol. 2012;2012:219625.

32. Chen TY, Chi KH, Wang JS, Chien CL, Lin WW. Reactive oxygen species are involved in FasL-induced caspase-independent cell death and inflammatory responses. Free Radic Biol Med. 2009;46:643-55.

33. Martinet W, De Meyer GR, Timmermans JP, Herman AG, Kockx MM. Macrophages but not smooth muscle cells undergo benzyloxycarbonylVal-Ala-DL-Asp(O-Methyl)-fluoromethylketone-induced nonapoptotic cell death depending on receptor-interacting protein 1 expression: implications for the stabilization of macrophage-rich atherosclerotic plaques. J Pharmacol Exp Ther. 2006;317:1356-64.

34. Xu Y, Kim SO, Li Y, Han J. Autophagy contributes to caspase-independent macrophage cell death. J Biol Chem. 2006;281:19179-87.

35. Ye YC, Wang HJ, Yu L, Tashiro S, Onodera S, Ikejima T. RIP1-mediated mitochondrial dysfunction and ROS production contributed to tumor necrosis factor alpha-induced $L 929$ cell necroptosis and autophagy. Int Immunopharmacol. 2012;14:674-82.

36. Robinson N, McComb S, Mulligan R, Dudani R, Krishnan L, Sad S. Type I interferon induces necroptosis in macrophages during infection with Salmonella enterica serovar Typhimurium. Nat Immunol. 2012;13:954-62.

37. Acuna UM, Wittwer J, Ayers S, Pearce CJ, Oberlies NH, EJ DEB. Effects of (5Z)-7-oxozeaenol on MDA-MB-231 breast cancer cells. Anticancer Res. 2012;32:2415-21

38. Acuna UM, Wittwer J, Ayers S, Pearce CJ, Oberlies NH, EJ DEB. Effects of (5Z)-7-oxozeaenol on the oxidative pathway of cancer cells. Anticancer Res. 2012;32:2665-71

39. Fan $Y$, Cheng J, Vasudevan SA, Patel RH, Liang $L$, Xu X, et al. TAK1 inhibitor 5Z-7-oxozeaenol sensitizes neuroblastoma to chemotherapy. Apoptosis. 2013:18:1224-34.

40. Omori E, Matsumoto K, Zhu S, Smart RC, Ninomiya-Tsuji J. Ablation of TAK1 upregulates reactive oxygen species and selectively kills tumor cells. Cancer Res. 2010;70:8417-25.
41. Buglio D, Palakurthi S, Byth K, Vega F, Toader D, Saeh J, et al. Essential role of TAK1 in regulating mantle cell lymphoma survival. Blood. 2012;120:347-55.

42. Singh A, Sweeney MF, Yu M, Burger A, Greninger P, Benes C, et al. TAK1 inhibition promotes apoptosis in KRAS-dependent colon cancers. Cell. 2012;148:639-50.

43. Byron SA, Loch DC, Wellens CL, Wortmann A, Wu J, Wang J, et al. Sensitivity to the MEK inhibitor E6201 in melanoma cells is associated with mutant BRAF and wildtype PTEN status. Mol Cancer. 2012;11:75.

44. Muramoto K, Goto M, Inoue Y, Ishii N, Chiba K, Kuboi Y, et al. E6201, a novel kinase inhibitor of mitogen-activated protein kinase/extracellular signalregulated kinase kinase-1 and mitogen-activated protein kinase/extracellular signal-regulated kinase kinase kinase-1: in vivo effects on cutaneous inflammatory responses by topical administration. J Pharmacol Exp Ther. 2010;335:23-31.

45. Shen Y, Boivin R, Yoneda N, Du H, Schiller S, Matsushima T, et al. Discovery of anti-inflammatory clinical candidate E6201, inspired from resorcylic lactone LL-Z1640-2. III Bioorg Med Chem Lett. 2010;20:3155-7.

46. Ridder DA, Schwaninger M. TAK1 inhibition for treatment of cerebral ischemia. Exp Neurol. 2013;239:68-72.

47. White BJ, Tarabishy S, Venna VR, Manwani B, Benashski S, McCullough LD, et al. Protection from cerebral ischemia by inhibition of TGFbeta-activated kinase. Exp Neurol. 2012;237:238-45.

48. Zhang D, Hu Y, Sun Q, Zhao J, Cong Z, Liu H, et al. Inhibition of transforming growth factor beta-activated kinase 1 confers neuroprotection after traumatic brain injury in rats. Neuroscience. 2013;238:209-17.

49. Wynn TA, Chawla A, Pollard JW. Macrophage biology in development, homeostasis and disease. Nature. 2013:496:445-55.

50. Ajibade AA, Wang Q, Cui J, Zou J, Xia X, Wang M, et al. TAK1 negatively regulates NF-kappaB and p38 MAP kinase activation in Gr-1 + CD11b + neutrophils. Immunity. 2012;36:43-54.

51. Lamothe B, Lai $Y$, Hur L, Orozco NM, Wang J, Campos AD, et al. Deletion of TAK1 in the myeloid lineage results in the spontaneous development of myelomonocytic leukemia in mice. PLoS One. 2012;7, e51228.

52. Dondelinger $Y$, Aguileta MA, Goossens V, Dubuisson C, Grootjans S, Dejardin E, et al. RIPK3 contributes to TNFR1-mediated RIPK1 kinase-dependent apoptosis in conditions of CIAP1/2 depletion or TAK1 kinase inhibition. Cell Death Differ. 2013;20:1381-92.

53. Kajino-Sakamoto R, Omori E, Nighot PK, Blikslager AT, Matsumoto K, Ninomiya-Tsuji J. TGF-beta-activated kinase 1 signaling maintains intestinal integrity by preventing accumulation of reactive oxygen species in the intestinal epithelium. J Immunol. 2010;185:4729-37.

54. Omori E, Morioka S, Matsumoto K, Ninomiya-Tsuji J. TAK1 regulates reactive oxygen species and cell death in keratinocytes, which is essential for skin integrity. J Biol Chem. 2008;283:26161-8.

55. Meylan E, Burns K, Hofmann K, Blancheteau V, Martinon F, Kelliher M, et al. RIP1 is an essential mediator of Toll-like receptor 3-induced NF-kappa B activation. Nat Immunol. 2004;5:503-7.

56. Davis CW, Hawkins BJ, Ramasamy S, Irrinki KM, Cameron BA, Islam K, et al. Nitration of the mitochondrial complex I subunit NDUFB8 elicits RIP1- and RIP3-mediated necrosis. Free Radic Biol Med. 2010;48:306-17.

57. Chen SY, Chiu LY, Maa MC, Wang JS, Chien CL, Lin WW. zVAD-induced autophagic cell death requires C-Src-dependent ERK and JNK activation and reactive oxygen species generation. Autophagy. 2011;7:217-28.

58. Lartigue L, Kushnareva Y, Seong Y, Lin H, Faustin B, Newmeyer DD. Caspaseindependent mitochondrial cell death results from loss of respiration, not cytotoxic protein release. Mol Biol Cell. 2009;20:4871-84.

\section{Submit your next manuscript to BioMed Central and take full advantage of:}

- Convenient online submission

- Thorough peer review

- No space constraints or color figure charges

- Immediate publication on acceptance

- Inclusion in PubMed, CAS, Scopus and Google Scholar

- Research which is freely available for redistribution 